\title{
2001064800
}

AIAA-2001-1587

\section{VIBROACOUSTIC MODEL VALIDATION FOR A CURVED HONEYCOMB COMPOSITE PANEL}

\author{
Ralph D. Buehrle* and Jay H. Robinson ${ }^{\dagger}$ \\ NASA Langley Research Center, Hampton, Virginia \\ and \\ Ferdinand W. Grosveld $¥$ \\ Lockheed Martin Engineering and Sciences, Hampton, Virginia
}

\begin{abstract}
Finite element and boundary element models are developed to investigate the vibroacoustic response of a curved honeycomb composite sidewall panel. Results from vibroacoustic tests conducted in the NASA Langley Structural Acoustic Loads and Transmission facility are used to validate the numerical predictions. The sidewall panel is constructed from a flexible honeycomb core sandwiched between carbon fiber reinforced composite laminate face sheets. This type of construction is being used in the development of an all-composite aircraft fuselage. In contrast to conventional rib-stiffened aircraft fuselage structures, the composite panel has nominally uniform thickness resulting in a uniform distribution of mass and stiffness. Due to differences in the mass and stiffness distribution, the noise transmission mechanisms for the composite panel are expected to be substantially different from those of a conventional rib-stiffened structure. The development of accurate vibroacoustic models will aide in the understanding of the dominant noise transmission mechanisms and enable optimization studies to be performed that will determine the most beneficial noise control treatments. Finite element and boundary element models of the sidewall panel are described. Vibroacoustic response predictions are presented for forced vibration input and the results are compared with experimental data.
\end{abstract}

*Aerospace Engineer, Structural Acoustics Branch

${ }^{\dagger}$ Aerospace Engineer, Structural Acoustics Branch

¥Aerospace Engineering Manager, Associate Fellow

Copyright $@ 2001$ by the American Institute of Aeronautics and Astronautics, Inc. No copyright is asserted in the United States under Title 17, U.S. Code. The U.S. Government has a royaltyfree license to exercise all rights under the copyright claimed herein for Government Purposes. All other rights are reserved by the copyright owner.
INTRODUCTION

Improved numerical modeling techniques for midfrequency vibroacoustic predictions are required for design optimization and noise control applications in the transportation industries. In response to this need, recent work at NASA Langley Research Center ${ }^{1}$ has focused on the validation of finite element based models of conventional aluminum aircraft fuselage structures for dynamic response predictions into the kiloHertz region. In future aircraft designs, trade studies between conventional aluminum aircraft fuselage construction and composite fuselage construction will need to be addressed. Composite materials offer the potential for a significant weight advantage, while maintaining strength and fatigue properties. In order to preserve the weight advantage of composites, the noise transmission characteristics need to be examined in the early design phases to avoid weight penalties associated with add-on noise control treatments. Validated numerical modeling techniques for midfrequency vibroacoustic response predictions will enable acoustic requirements to be incorporated into the early design phases.

Conventional aluminum aircraft fuselage construction consists of a thin-aluminum skin with longitudinal and circumferential stiffeners. New all-composite aircraft fuselage designs are being developed ${ }^{2}$ with a flexible honeycomb core sandwiched between carbon fiber reinforced composite laminate face sheets. The honeycomb composite fuselage sidewalls have nominally uniform thickness resulting in a uniform distribution of mass and stiffness. Therefore, the noise transmission mechanisms for a honeycomb composite sidewall are expected to be substantially different from those of a conventional rib-stiffened structure. In a joint program between the United States Air Force and NASA, Grosveld and Reed ${ }^{3}$ conducted sound transmission loss experiments on several curved aircraft type panels 
constructed of aluminum and honeycomb composite. In this study it was found that the uniform distribution of the mass for a honeycomb composite panel resulted in increased transmission loss values at higher frequencies as compared to a conventional rib-stiffened aluminum fuselage panel with comparable mass. Additional numerical and experimental studies are needed to further characterize the dominant noise transmission mechanisms for honeycomb composite structures.

This work focuses on the development and validation of finite element and boundary element models for the prediction of the vibroacoustic response of a curved honeycomb composite sidewall panel. The models were developed based on the physical dimensions and material properties of the structure as defined in the manufacturing specifications. Initial validation of the finite element model is based on comparisons with modal test data. Vibration and acoustic response measurements with the panel installed in the NASA Langley Structural Acoustic Loads and Transmission (SALT) Facility ${ }^{4}$ provide the data for validation of the vibroacoustic predictions. This paper describes the test facility, hardware, numerical models, and validation tests. Comparisons between numerical and experimental vibroacoustic data are presented for point force excitation.

\section{TEST FACILITY}

The Structural Acoustic Loads and Transmission (SALT) Facility ${ }^{4}$ located at the NASA Langley Research Center consists of an anechoic chamber, a reverberation chamber, and a shared transmission loss (TL) window. The anechoic chamber has a volume of 337 cubic meters. Interior dimensions of the anechoic chamber, measured from wedge tip to wedge tip, are 4.57 meters in height, 7.65 meters in width, and 9.63 meters in length. The reverberation chamber has approximate dimensions of 4.5 meters in height, 6.5 meters in width, and 9.5 meters in length for a volume of 278 cubic meters. A shared TL window accommodates test structures of up to 1.41 meters by 1.41 meters. For the tests on the honeycomb composite panel, the SALT facility was setup as a transmission loss suite with the reverberation chamber on the source side of the panel, corresponding to the aircraft exterior, and the anechoic chamber on the receiver side, corresponding to the aircraft interior.

\section{HARDWARE DESCRIPTION}

For the purposes of this study, a curved sidewall panel was constructed from a flexible nomex honeycomb core sandwiched between carbon fiber reinforced composite laminate face sheets. Figure 1 shows the honeycomb composite panel resting on foam wedges in the anechoic room of the SALT facility. As shown in the figure, the panel edges were unfinished leaving the honeycomb core exposed. The 2.06-centimeter $(\mathrm{cm})$ thick panel has a length of 1.39-meter $(\mathrm{m})$, an inner radius of $1.04 \mathrm{~m}$, and covers an arc of approximately 82 degrees.

The panel is shown mounted in the transmission loss window of the SALT facility in Figure 2. This figure shows the exterior of the panel as seen from the reverberation chamber. The curvature of the test panel creates significant challenges for mounting in the flat transmission loss window. On the curved edges, $7.62-\mathrm{cm}$ thick end caps were manufactured from medium density fiberboard to minimize the transmission of acoustic energy at these boundaries. Fiberboard framing was also manufactured for the straight edges of the panel. Channels were cut into the fiberboard frame to accommodate the composite panel. The edges of the panel were fit into the channel and a compression fit was maintained using plastic tubing. The plastic tubing contacts the interior and exterior laminate surfaces at approximately 0.48 $\mathrm{cm}$ from the panel edge. This provides the only contact between the mounting hardware and the test panel. The motivation for this clamping mechanism was to provide a uniform boundary condition along the edges of the panel for numerical modeling purposes and to prevent acoustic leaks during testing.

\section{NUMERICAL MODELS}

Finite element and boundary element models were developed from the geometric, material and structural properties of the curved honeycomb composite sidewall panel. The finite element model was developed using MSC/PATRAN and analyzed using MSC/NASTRAN. As shown in Figure 3, the panel was meshed with 42 elements along the length by 42 elements along the arc. Solid elements were used to model the honeycomb core. The face sheets were modeled using linear plate elements with appropriate laminate properties. To validate the finite element model, a normal mode analysis was performed for free-free boundary conditions and the results were compared with modal test data under simulated free-free boundary conditions. 
After installation of the panel in the SALT facility, a second normal mode analysis was performed and the results were compared with modal test data for the as-installed configuration. Grounded springs were introduced at the nodes along the perimeter of the finite element model to simulate the boundary conditions of the test panel. The stiffness of the boundary condition springs was adjusted in order to obtain agreement between the predicted and measured modal frequencies.

The validated finite element model was used to predict the surface velocities for a point force excitation. Damping values for the forced response analysis were determined from the measured modal data. Surface velocities on the interior of the panel were predicted over the 100 to $600 \mathrm{~Hz}$ frequency range using a modal solution including all modes up to $3000 \mathrm{~Hz}$. This data provides the required boundary condition input for the boundary element predictions of the radiated sound pressure.

The boundary element model was developed using COMET/Nision and analyzed using COMET/Acoustics. The initial boundary element mesh for the panel surface contained 16 elements along the length by 16 elements along the arc. A refined 32 - by 32-element mesh was later developed to provide better spatial definition for the high frequency dynamic response. Figure 4 shows the boundary element model with the refined panel mesh. In order to compare with the test results, the mounting fixture and a portion of the transmission loss window were included in the boundary element model and treated as rigid surfaces. For a direct boundary element analysis, the model must be fully enclosed with no free edges. Therefore, the edges of the transmission loss window were enclosed.

A direct boundary element analysis was used. For this uncoupled solution process, the velocities on the panel surface were first determined from a finite element based frequency response analysis. COMET/Vision was then used to interpolate from the frequency response results to the velocity boundary conditions. The acoustic response of the panel for the prescribed velocity boundary conditions was then predicted for radiation into the free field. Sound pressure levels were computed for both a near field pressure recovery grid, 9.8 centimeters from the panel surface, and a far field pressure recovery grid, 3.7 meters from the center of curvature for the panel.
Acoustic predictions were also obtained using measured frequency response function data to obtain the velocity boundary conditions for the boundary element model. The frequency response function data was obtained at the boundary element node points using a scanning laser doppler vibrometer while an electrodynamic shaker excited the panel. The normalized nodal velocities were averaged to obtain element level velocity boundary conditions. The acoustic response of the panel for the measured velocity boundary conditions was then predicted for radiation into the free field.

\section{MODAL TESTS}

To validate the finite element model, modal tests were performed on the composite sidewall panel with two different boundary conditions. The first test was conducted with the panel supported on four foam blocks to simulate free-free edge conditions. A second modal test was conducted with the panel installed in the SALT facility.

The data acquisition and analysis was similar for both modal tests. Impact input was used to excite the panel and the response was measured with three reference accelerometers. A measurement grid of 9 points along the straight edge by 11 points along the arc was used. Inertance frequency response functions were measured and averaged for five impacts at each of the grid locations. Data were acquired over a 0 to $500 \mathrm{~Hz}$ frequency range for the free-free test and 0 to 625 $\mathrm{Hz}$ range for the installed configuration. The polyreference curvefitter in the MTS/IDEAS software was used to determine the modal properties from the frequency response functions.

\section{VIBROACOUSTIC TESTS}

Vibration and acoustic response measurements were acquired for point force excitation from a shaker. Figure 5 shows the interior of the panel from the anechoic chamber. From this view, the shaker location was in the lower left quadrant. This excitation location was used for all of the vibroacoustic tests and analyses presented in this paper. In Figure 5, a vertical array of sixteen equally spaced microphones is also shown. The array was mounted on a rotational mechanism that allowed for automated positioning of the microphones over an arc of eighty degrees. For this series of tests, the microphones were placed at a distance of $9.8 \mathrm{~cm}$ from the panel surface and used to measure the near field sound pressure over 80 degrees in five degree increments. 
Far field sound pressure measurements were acquired at four locations as shown in Figure 6. The center microphone was set at the height of the panel center and at a distance of 3.7 meters from the center of curvature for the panel. The three remaining microphones were set at a distance of 1.2 meters in the vertical and horizontal directions from the center microphone. In addition to the microphones, the driving point force and panel accelerations at five locations on the panel were measured.

A random shaker input was used to excite the panel over a $1000 \mathrm{~Hz}$ frequency range. For each angular position of the near field microphone array, data was acquired for 100 averages, using a Hanning window, with $1000 \mathrm{~Hz}$ frequency range and a resolution of $1.25 \mathrm{~Hz}$. Spectra, coherence and frequency response function data were measured with the force input as the reference.

In addition to the vibroacoustic measurements, the panel and shaker setup was used to measure the surface velocity data required for the boundary element analysis. A scanning laser doppler vibrometer was used to measure the surface velocity at each of the boundary element nodes for the 16- by 16-element mesh due to a psuedo random shaker input. To minimize the curvature effects, the panel was divided into four vertical segments and separate scans were performed on each. The laser was aligned with the center of the panel segment and the distance to the panel was adjusted to optimize measurement quality. Surface roughness on the interior of the composite panel caused some degradation in the data quality. Frequency response function data were obtained between the velocity response and input force over a $1000 \mathrm{~Hz}$ frequency range with a $1.25 \mathrm{~Hz}$ resolution. Since the psuedo random input was periodic in the measurement window, no window was applied to the data and only five averages were used. The normalized velocity data from the four segments were combined into a single data set and used to determine the element level boundary conditions needed for the boundary element analysis.

\section{RESULTS AND DISCUSSION}

The finite element predictions of the free-free modal frequencies of the composite sidewall panel are compared with the measured results in Table 1. In Table 1, each mode is described by the number of nodal lines, $i$, along the straight edge and, $j$, along the arc. Figure 7 shows the measured $i=3, j=1$ mode shape at $156.7 \mathrm{~Hz}$. The white and black represent motion that is 180 degrees out of phase. As shown in Table 1, predicted natural frequencies are within $2.6 \%$ of the measured values. This provided confidence that the finite element model accurately represented the physical structure. Modal damping estimates for this configuration ranged from 0.4 to $1.8 \%$.

Grounded springs were used to model the boundary conditions of the panel installed in the SALT facility. The stiffness of the springs was adjusted to obtain agreement between the predicted and measured modal frequencies for the installed configuration. Predicted natural frequencies with the adjusted boundary condition springs are compared with measured results in Table 2. For consistency with the free-free results, each mode is described by the number of nodal lines, $i$, along the straight edge and, j, along the arc. Figure 8 shows the measured $i=2, j=1$ mode at $301.6 \mathrm{~Hz}$. As shown in Table 2, the predicted results are within $3.1 \%$ of the measured values. It should be noted that not all modes were identified during the modal tests. This may be associated with the selection of reference positions for the modal test being near node lines for a particular mode. Also, in the installed configuration, the panel response was more heavily damped making it difficult to separate closely spaced modes. Estimated modal damping values ranged from 1.6 to $4.2 \%$.

The validated finite element model was used to predict the vibration response on the interior surface of the panel for point force excitation. The frequency response analysis used a modal superposition approach including all modes through $3000 \mathrm{~Hz}$. Damping values for the analysis were based on the measured modal damping. Figure 9 shows a comparison between the predicted and measured driving point inertance frequency response function. This is the ratio of the acceleration response at the excitation location to the excitation force. The predicted and measured frequency response functions are in good agreement over the 200 to $600 \mathrm{~Hz}$ range. In the 100 to $200 \mathrm{~Hz}$ range, the predicted response appears more lightly damped. To improve the predictions, the modal damping in this low frequency region could be increased in the finite element model.

The measured surface velocity at a frequency of $202.5 \mathrm{~Hz}$ is shown in Figure 10. This is a view of

4 
the interior of the panel from the anechoic chamber. The frequency of $202.5 \mathrm{~Hz}$ was selected for comparison since it corresponds to the first dominant peak in the far field sound pressure. Figure 11 shows the corresponding finite element based prediction of the surface velocity at $202.5 \mathrm{~Hz}$. The spatial characteristics for the measured and predicted surface velocities are in good agreement.

An effort to import and interpolate the velocity boundary conditions from the finite element data was unsuccessful. The boundary element program was not readily able to interpolate and map the three-dimensional, solid, finite element results onto the two-dimensional boundary element mesh. Figure 12 shows the resulting interpolated boundary condition for $202.5 \mathrm{~Hz}$. This velocity boundary condition data does not agree with the original finite element surface velocity predictions shown in Figure 11. Efforts are underway to reformat the finite element results to a suitable format for the mapping and interpolation program. Due to this problem, comparisons of the radiated sound pressure are not provided for the finite element based surface velocity data.

The measured surface velocities were mapped to the original 16- by 16 -element mesh of the panel using a user-generated program. Figure 13 shows the panel portion of the boundary element model with the measured velocity boundary conditions at $202.5 \mathrm{~Hz}$. This is consistent with the original measured surface velocities shown in Figure 10. Based on the measured velocity boundary conditions, boundary element predictions of the radiated sound pressure were made. Figure 14 shows the measured and predicted far field sound pressure for the microphone that is aligned with the panel center. This microphone is at a distance of 3.7 meters from the panel center of curvature. Good agreement between the measured and predicted sound pressures is obtained. Further evaluation of the predicted near field and far field pressures are underway.

\section{SUMMARY AND CONCLUSIONS}

The finite element model of the curved honeycomb composite sidewall panel was validated with modal test data. Excellent agreement was obtained with predicted natural frequencies within $3.1 \%$ of the measured values for both the free-free and installed boundary conditions. The predicted and measured driving point frequency response functions were in good agreement over the 200 to $600 \mathrm{~Hz}$ frequency range.
The finite element predictions of the panel surface velocity are in good agreement with the measured spatial characteristics. Efforts are currently underway to import and interpolate the velocity boundary conditions from the finite element data. The imported velocity boundary condition data will be used as input to the boundary element solution for the radiated sound pressure.

Boundary element predictions of the radiated sound pressure using measured velocity boundary condition data show good agreement for the far field microphone that is aligned with the panel center. Additional evaluation of the predicted near field and far field pressures is underway.

\section{Reference:}

1. Buehrle, R. D., Fleming, G. A., Pappa, R. S., and Grosveld, F. W., "Finite Element Model Development and Validation for Aircraft Fuselage Structures," Sound and Vibration Magazine, January, 2001.

2. Bokulich, Frank, "Raytheon's Premier I Makes First Flight", Aerospace Engineering, April 1999.

3. Grosveld, F. W., and Reed, S. A., "Sound Transmission of Integrally Damped, Curved Panels," Proceedings of InterNoise 89, Newport Beach, California, December 1989.

4. Grosveld, F. W., "Calibration of the Structural Acoustic Loads and Transmission Facility at NASA Langley Research Center," Proceedings of InterNoise 99, Fort Lauderdale, Florida, December 1999. 
Table 1. Analytical and measured modal frequencies for the honeycomb composite panel with free-free boundary conditions.

\begin{tabular}{|c|c|c|c|}
\hline Analysis & Measured & $\begin{array}{c}\text { Mode } \\
\text { Description }\end{array}$ & $\begin{array}{c}\text { Analysis } \\
\text { Measured }\end{array}$ \\
\hline Frequencs & Frequency & Node lines, i, & Difference \\
\hline$[\mathrm{Hz}]$ & {$[\mathrm{Hz}]$} & $\begin{array}{c}\text { in vertical and, } \\
j, \text { on arc }\end{array}$ & {$[\%]$} \\
\hline 34.2 & 34.5 & $i=1, j=1$ & -0.9 \\
\hline 53.1 & 54.4 & $i=2, j=0$ & -2.4 \\
\hline 79.2 & 77.2 & $i=2, j=1$ & 2.6 \\
\hline 134.0 & 132.9 & $i=3, j=0$ & 0.8 \\
\hline 156.7 & 153.6 & $i=3, j=1$ & 2.0 \\
\hline 159.7 & 159.8 & $i=2, j=2$ & -0.1 \\
\hline 166.4 & 164.6 & $i=1, j=2$ & 1.1 \\
\hline 233.1 & 230.0 & $\mathrm{i}=4, \mathrm{j}=0$ & 1.3 \\
\hline 249.4 & 245.6 & $i=4, j=1$ & 1.5 \\
\hline 280.5 & 278.8 & $i=3, j=2$ & 0.6 \\
\hline 294.4 & 295.3 & $i=2, j=3$ & -0.3 \\
\hline 294.4 & 300.0 & $i=1, j=3$ & -1.9 \\
\hline 320.3 & 318.5 & $i=4, j=2$ & 0.6 \\
\hline 335.8 & 334.5 & $i=5, j=0$ & 0.4 \\
\hline 352.0 & 350.8 & $\mathrm{i}=5, \mathrm{j}=1$ & 0.3 \\
\hline 402.6 & 402.6 & $\mathrm{il}=5, \mathrm{j}=3$ & 0.0 \\
\hline
\end{tabular}

Table 2. Analytical and measured modal frequencies for the honeycomb composite panel installed in SALT.

\begin{tabular}{|c|c|c|c|}
\hline Analytical & Measured & $\begin{array}{c}\text { Mode } \\
\text { Description }\end{array}$ & $\begin{array}{l}\text { Analytical } \\
\text { Measured }\end{array}$ \\
\hline Frequency & Frequency & Node lines, $\mathrm{i}$, & Difference \\
\hline$[\mathrm{Hz}]$ & {$[\mathrm{Hz}]$} & $\begin{array}{c}\text { in vertical and, } \\
j, \text { on arc }\end{array}$ & {$[\%]$} \\
\hline 138.6 & 142.0 & $i=1, j=0$ & -2.4 \\
\hline 142.6 & - & $i=0, j=0$ & \\
\hline 201.2 & 205.6 & $i=2, j=0$ & -2.1 \\
\hline 274.7 & - & $i=1, j=1$ & \\
\hline 281.0 & - & $i=0, j=1$ & \\
\hline 289.9 & 299.1 & $i=3, j=0$ & -3.1 \\
\hline 298.3 & 301.6 & $i=2, j=1$ & -1.1 \\
\hline 355.2 & 356.7 & $i=3, j=1$ & -0.4 \\
\hline 385.8 & 392.2 & $i=4, j=0$ & -1.6 \\
\hline 426.0 & - & $i=2, j=2$ & \\
\hline 433.9 & - & $i=1, j=2$ & \\
\hline 435.1 & 439.1 & $i=4, j=1$ & -0.9 \\
\hline 465.8 & 456.5 & $i=3, j=2$ & 2.0 \\
\hline 482.6 & 483.9 & $i=5, j=0$ & -0.3 \\
\hline 518.9 & 519.0 & $\mathrm{i}=4, \mathrm{j}=2$ & -0.0 \\
\hline 522.8 & 526.6 & $i=5, j=1$ & -0.7 \\
\hline
\end{tabular}

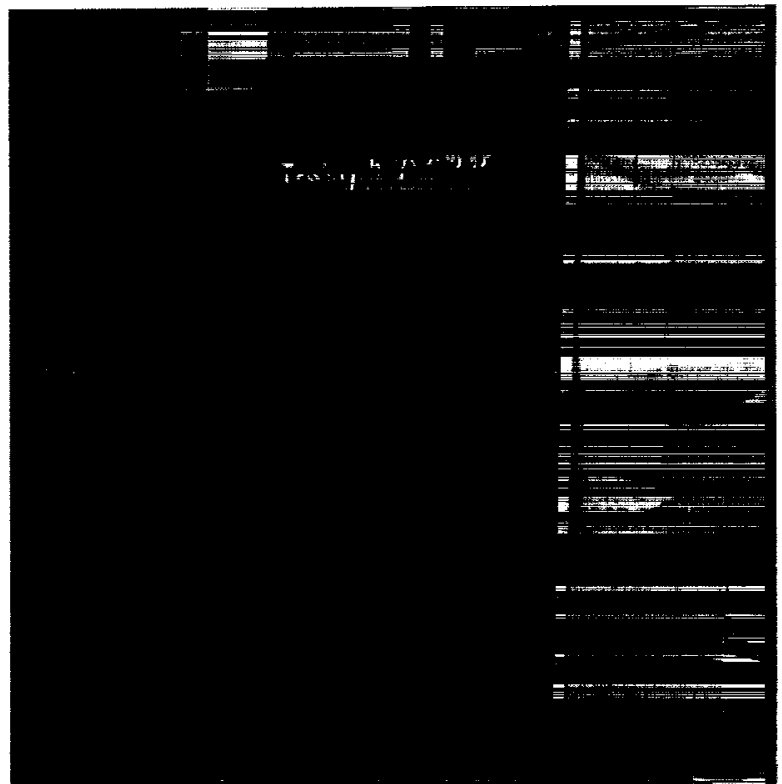

Figure 1. Honeycomb composite sidewall panel in the anechoic room of the SALT facility.

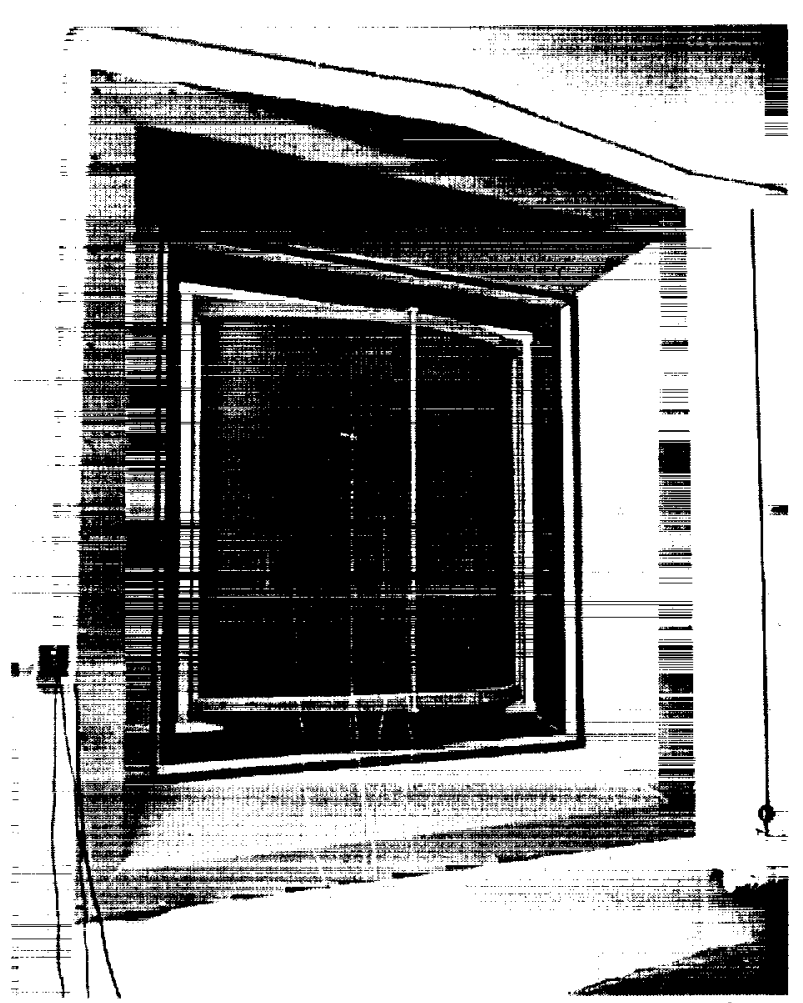

Figure 2. Curved honeycomb composite panel installed in the SALT transmission loss window. 

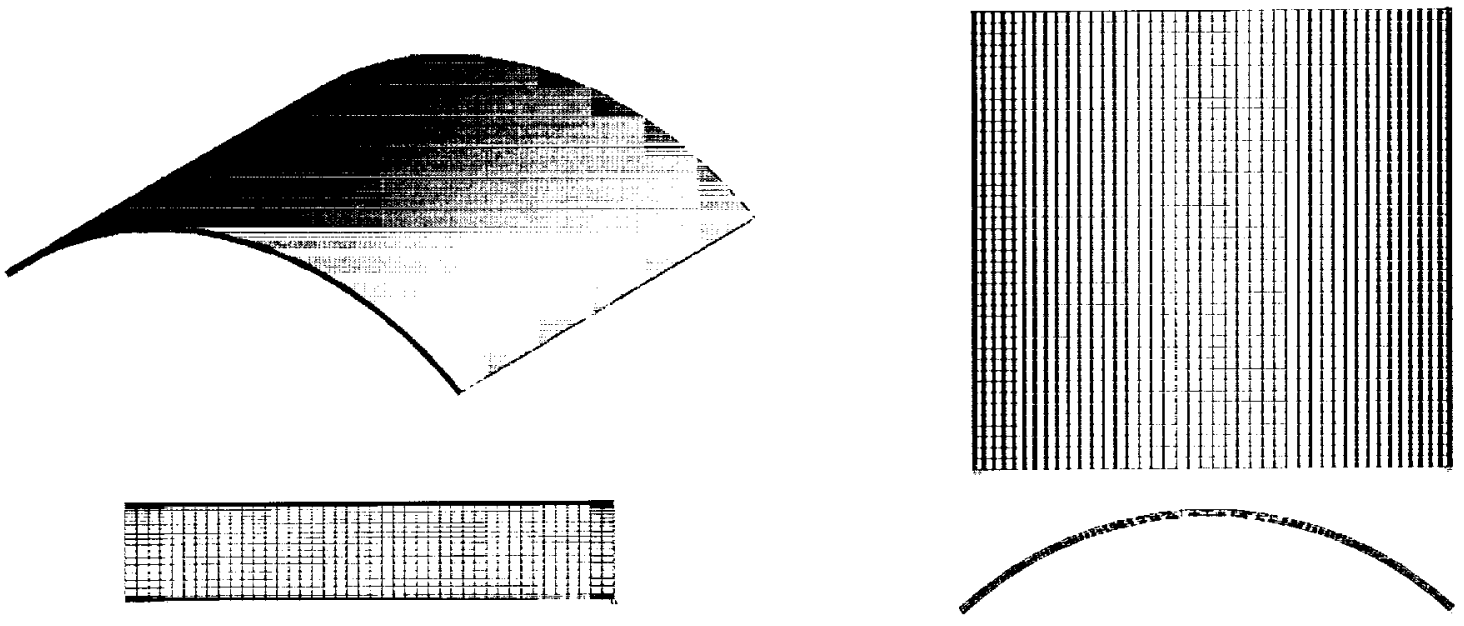

Figure 3. Finite element model of the curved honeycomb composite panel.
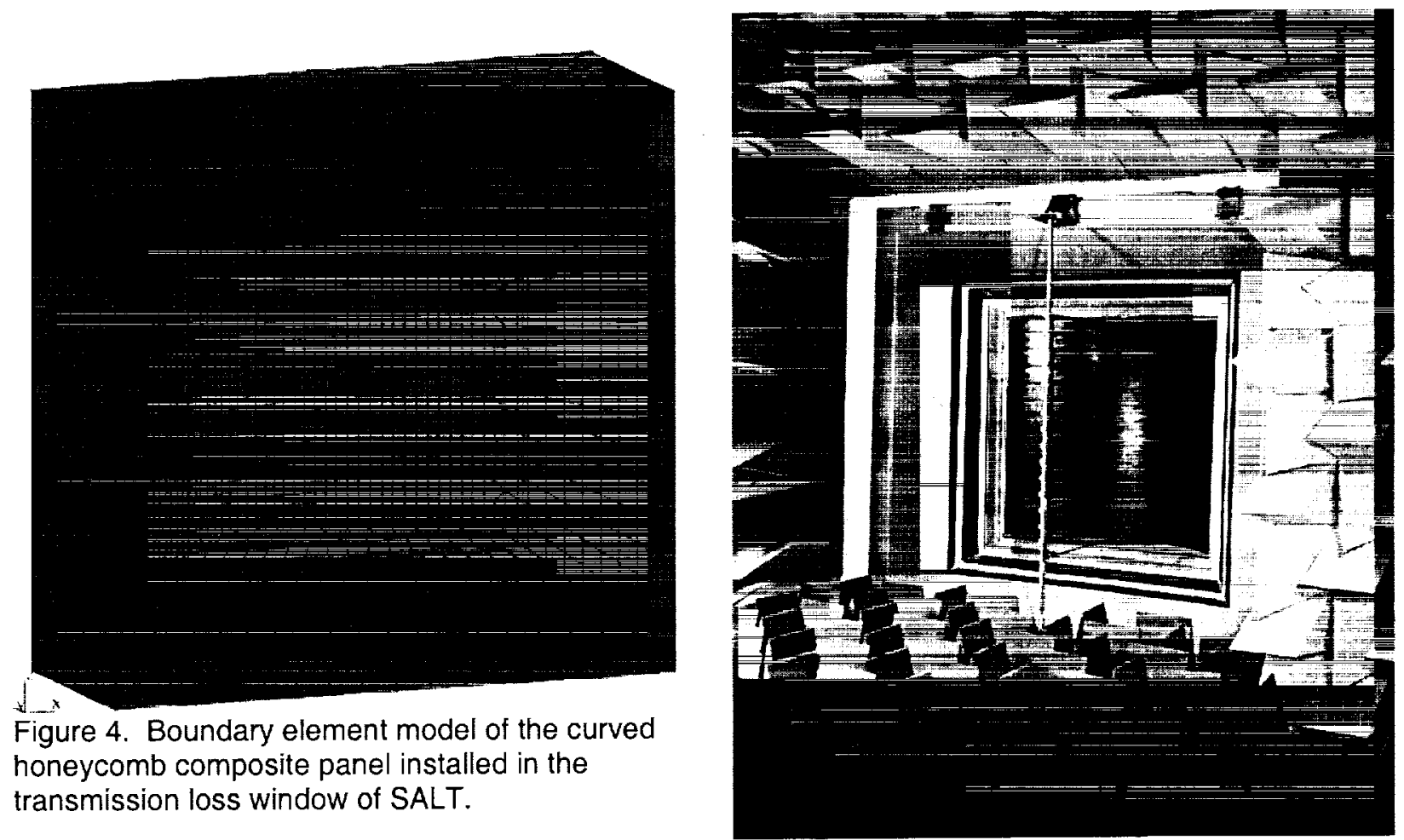

Figure 5. View of the vibroacoustic test setup for the curved honeycomb composite panel from the anechoic chamber of SALT. 


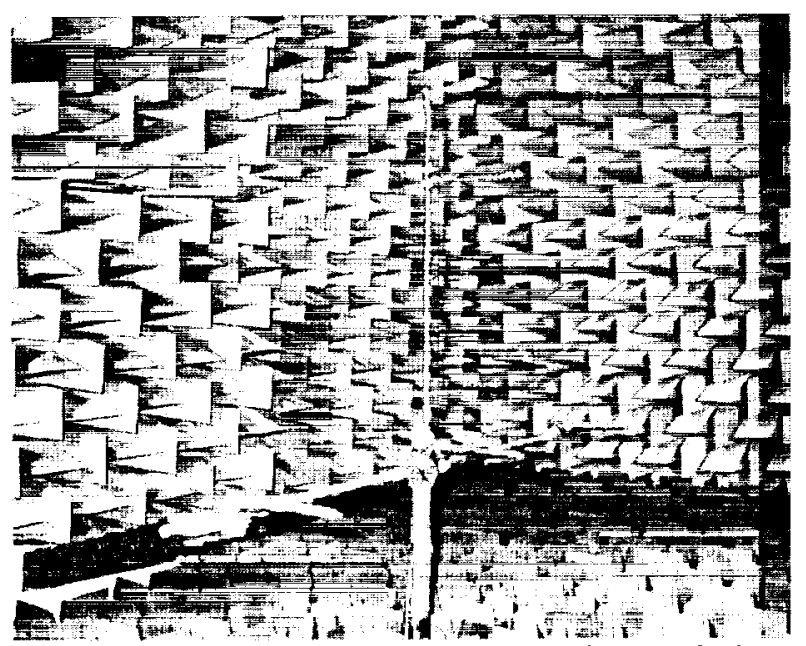

Figure 6. Far field microphone array in anechoic chamber of SALT.

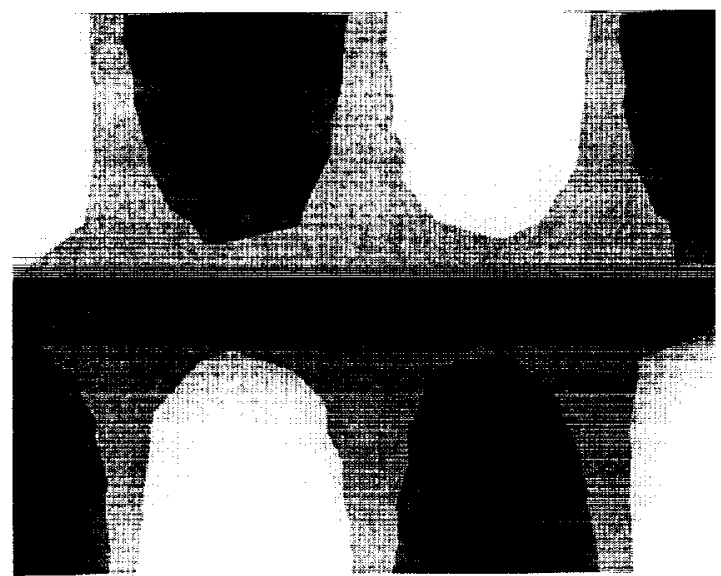

Figure 7. Measured $i=3, j=1$ mode shape at 156.7 $\mathrm{Hz}$ for the panel under free-free boundary conditions.

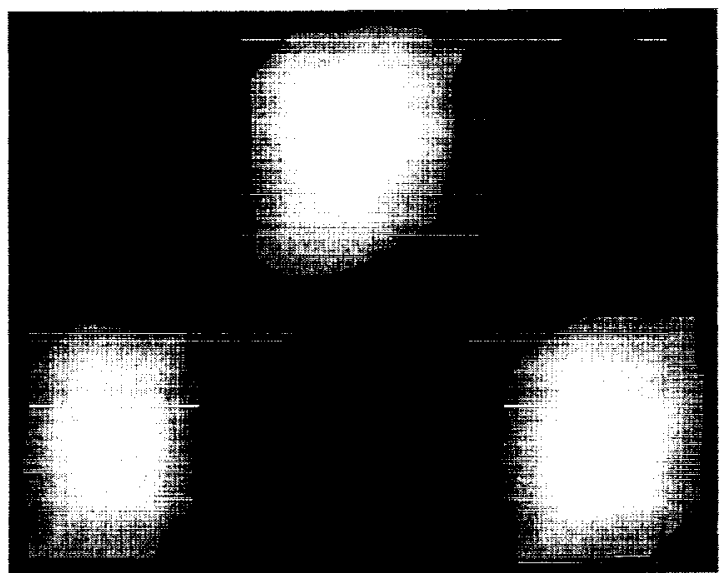

Figure 8. Measured $i=2, j=1$ mode shape at 301.6 $\mathrm{Hz}$ for the panel installed in SALT.

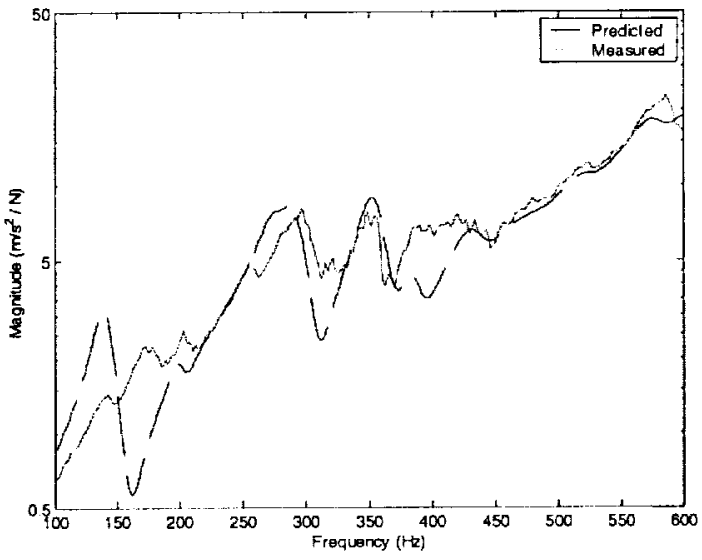

Figure 9. Measured and predicted driving point frequency response function magnitude.

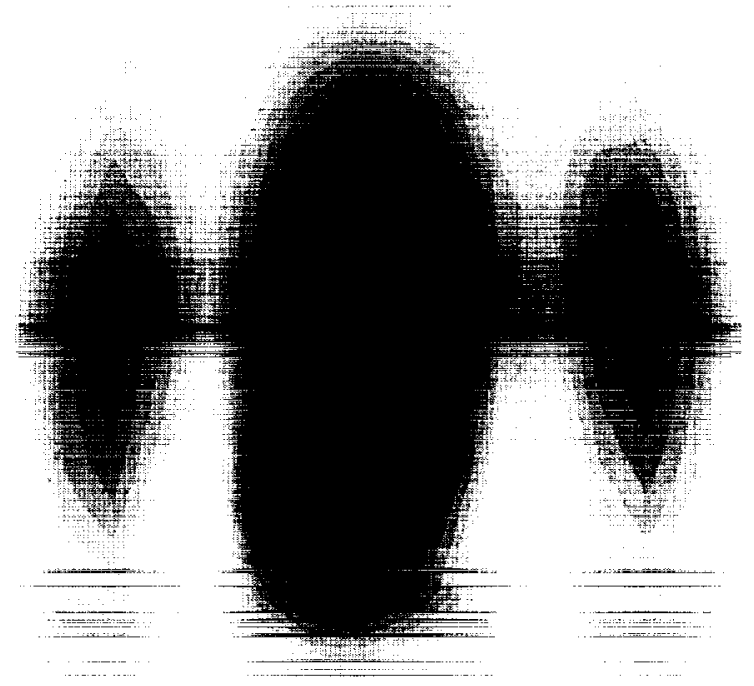

Figure 10. Measured velocity magnitude on the interior panel surface for a frequency of $202.5 \mathrm{~Hz}$. 

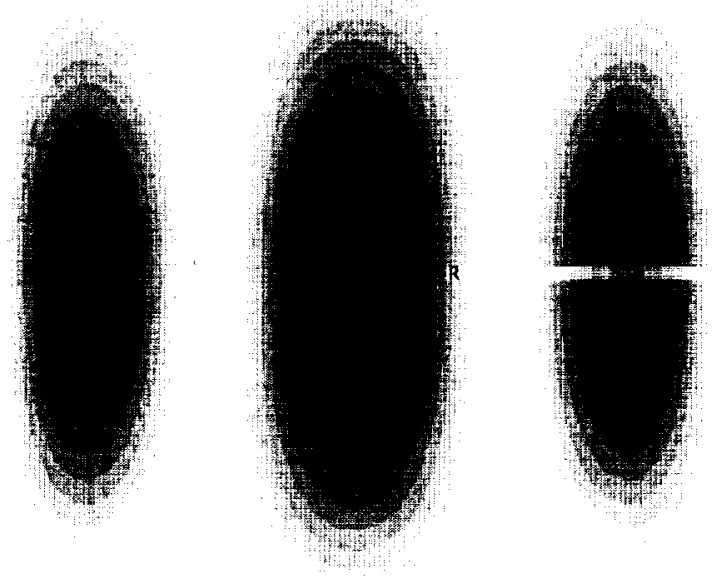

Figure 11. Finite element prediction of the velocity magnitude on the interior panel surface for a frequency of $202.5 \mathrm{~Hz}$.

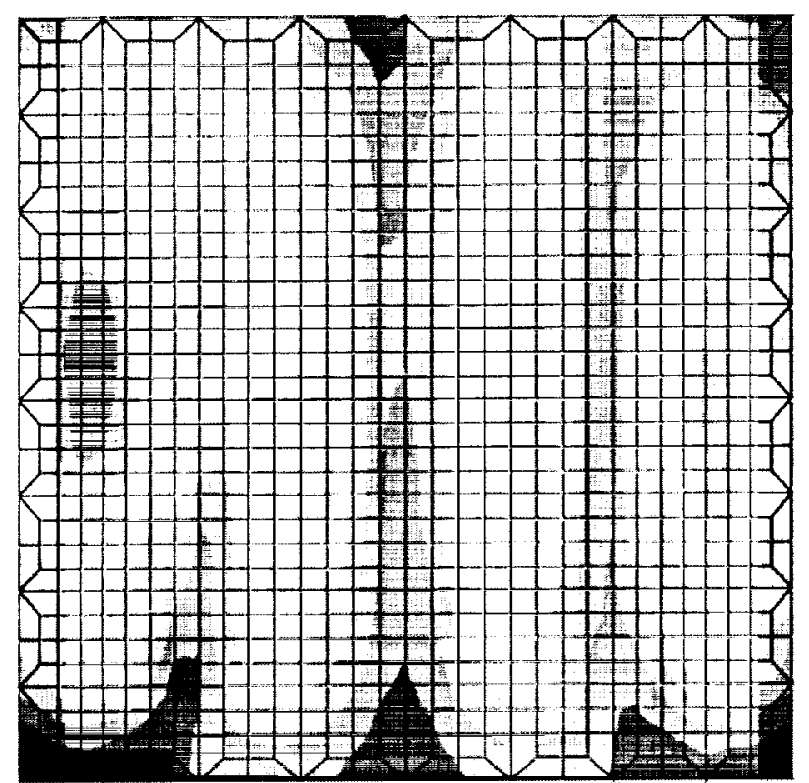

Figure 12. Interpolated finite element based velocity boundary condition for a frequency of $202.5 \mathrm{~Hz}$ shown applied to the refined mesh boundary element model.

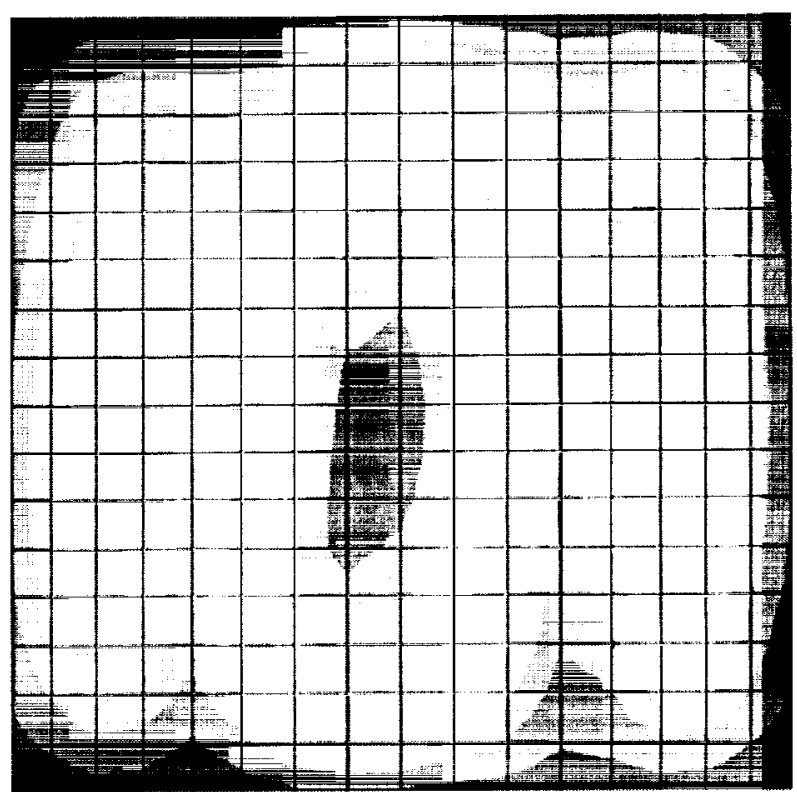

Figure 13. Measurement based velocity boundary condition for a frequency of $202.5 \mathrm{~Hz}$ shown applied to the boundary element model.

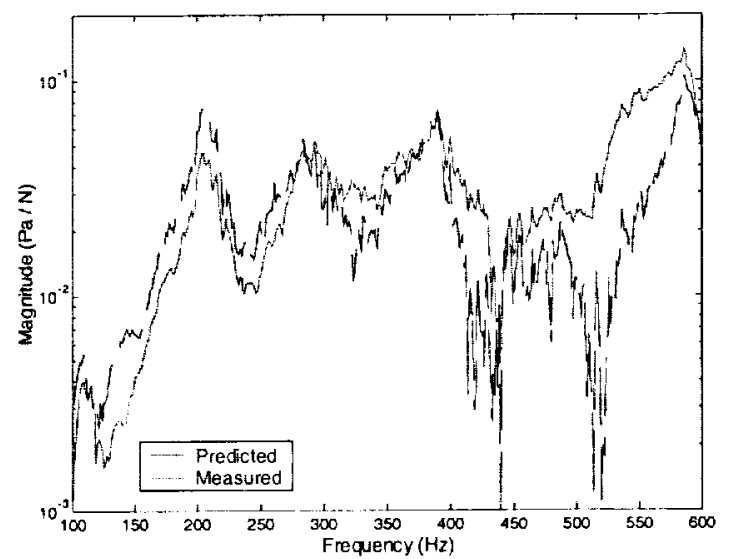

Figure 14. Measured and predicted sound pressure normalized by the input force for the far field microphone aligned with the panel center. 